Check for updates

Cite this: RSC Adv., 2018, 8, 36331

\title{
Synthesis, characterization and osteogenesis of phosphorylated methacrylamide chitosan hydrogels
}

\author{
Huishang Yang,$\dagger^{\mathrm{b}}$ Shenggui Chen, (D) $\dagger^{\mathrm{b}}$ Lei Liu, $^{\mathrm{b}}$ Chen Lai ${ }^{\mathrm{a}}$ and Xuetao Shi (D) *b
}

Phosphorylated biopolymers can induce mineralization, mimic the process of natural bone formation, and have the potential as scaffolds for bone tissue engineering. 2-Methacryloyloxyethyl phosphorylcholine (MPC), a low cytotoxicity phosphorus source, is mainly applied in vascularization and promoting blood compatibility and has been less researched for bone repair. In this study, phosphorylated methacrylamide chitosan (PMAC) hydrogel was prepared by mixing methacrylamide chitosan (MAC) and different mass of MPC with photoinitiator under UV irradiation. A series characterization tests showed that PMAC hydrogels were successful prepared and had a pretty good mineralization ability. Moreover, human fetal osteoblastic (hFOB) cells cultured on PMAC hydrogels exhibited not only highly viability but also the enhanced ALP activity and calcium deposition. The PMAC hydrogels have great potential in bone tissue engineering applications.

Received 23rd June 2018

Accepted 18th September 2018

DOI: $10.1039 / \mathrm{c} 8 \mathrm{ra0} 05378 \mathrm{~b}$

rsc.li/rsc-advances

There are generally two kinds of reagents used in the synthesis of phosphorylated biopolymers: skeleton phosphorylation and side group phosphorylation. Skeleton phosphorylation biopolymers are polymers themselves so they are not suitable to modify other polymeric materials. Side group phosphorylation biopolymers are mostly cytotoxic, oil-soluble, poor biological functionality and may not be suitable to be modified into biopolymer materials because of their toxic degradation products. Therefore, the key to the preparation of phosphorylated hydrogels is to find phosphorus sources with low cytotoxicity. 2Methacryloyloxyethyl phosphorylcholine (MPC), a low cytotoxicity phosphorus source, is mainly applied in vascularization and promoting blood compatibility ${ }^{28,29}$ and has less been researched for bone repair.

In this study, methacrylamide chitosan (MAC) was prepared by reaction of chitosan and methacrylic anhydride (MA) to graft carbon-carbon double bonds of methacrylic anhydride onto amino groups of chitosan, which provide the reaction point for the subsequent crosslinking reaction. ${ }^{30}$ Then MAC was reacted with 2-methacryloyloxyethyl phosphorylcholine (PMAC) with different mass fraction and photo-crosslinked by adding photoinitiator under UV irradiation to prepare phosphorylated methacrylamide chitosan (PMAC) hydrogels. Human fetal osteoblastic (hFOB) cells were cultured on the materials to evaluate biocompatibility and osteogenesis.

\section{Materials and method}

\subsection{Materials}

Methacrylic anhydride, 2-hydroxy-4'-(2-hydroxyethoxy)-2methylpropiophenone (Irgacure 2959), alizarin red S and 
sodium dodecyl sulfate (SDS) were purchased from SigmaAldrich (USA). Chitosan (CS, viscosity average molecular weigh: $47 \times 10^{4} \mathrm{~g} \mathrm{~mol}^{-1}$, degree of deacetylation $>95 \%$ ) was purchased from Aladdin (China). Dulbecco's modified Eagle's medium (DMEM)/F12 (DMEM-F12), phosphate-buffered saline (PBS), trypsin-EDTA, and foetal bovine serum (FBS) were obtained from Gibco (USA).

\subsection{Preparation of MAC}

A $1 \mathrm{wt} \%$ solution of chitosan was prepared by dissolving $1 \mathrm{~g}$ of chitosan $(\mu=200-400 \mathrm{mPa} s)$ in $99 \mathrm{~mL}$ deionized water, and then $1 \mathrm{~mL}$ acetic acid was added into the solution with constant string. $4 \mathrm{~mL}$ of methacrylic anhydride was added into the solution and stirred overnight at room temperature. Several dialysis bags were filled with 1/3-1/2 the solution and were put in deionized water for about 5 days with the water changed three times a day. Then the solution was transferred into glass culture dishes and lyophilized.

\subsection{Preparation of PMAC hydrogels}

Three different mass fraction PMAC hydrogels were prepared. Briefly, a 1 wt $\%$ solution of MAC was prepared by totally dissolved in deionized water with constant string. Different mass fraction MPC and $1 \mathrm{wt} \%$ cytocompatible photo-initiator Irgacure 2959 were added into the solution separately. ${ }^{\mathbf{1 3 , 2 0}}$ The mixture was transferred into 48-well plate with $250 \mu \mathrm{L}$ each hole by pipette. The plate was placed in ultrasonic cleaning machine for 10 minutes with $70 \%$ power to remove the air bubble. Then the plate was placed under UV-irradiation for 30 minutes to produce free radicals for irradiation and further initiate polymerization. The mass fraction ration of MAC/MPC was $1,0.5$ and 0.1 and named PMAC 1, PMAC 0.5 and PMAC 0.1, respectively. The PMAC hydrogels were immersed in deionized water to remove the unreacted monomer before characterization. ${ }^{\mathbf{1 2 , 1 8 , 2 6}}$

\subsection{Fourier transform infrared spectrometer (FITR)}

The FITR (CCR-1, Thermo-Nicolet, USA) was collected between 400 and $4000 \mathrm{~cm}^{-1}$ with a resolution of $2 \mathrm{~cm}^{-1}$ and was used to compare the structure differences among pure CS, MAC and different mass fraction of PMAC according to a modified published procedure.

\section{$2.5{ }^{1} \mathrm{H}$ NMR and ${ }^{31} \mathrm{P}$ NMR spectrometer}

${ }^{1} \mathrm{H}$ NMR and ${ }^{31} \mathrm{P}$ NMR were used to confirm the structure composition of CS, MAC and PMAC according to a modified published procedure. ${ }^{31}{ }^{1} \mathrm{H}$ and ${ }^{31} \mathrm{P}$ NMR spectra were obtained using a $400 \mathrm{MHz}$ spectrometer (AVANCE Digital 400, Bruker, Germany). The samples were dissolved in $\mathrm{D}_{2} \mathrm{O}$ with $1 \%$ acetic acid-d4 to a concentration of $10 \mathrm{mg} \mathrm{mL}^{-1}$.

\subsection{Thermogravimetric analysis (TG)}

TG was used to test the thermal stability of MAC and PMAC. Thermal stability of the synthesized materials was analysed in nitrogen atmosphere $\left(20 \mathrm{~mL} \min ^{-1}\right)$ using a synchronous thermal analyser (STA 449C, NETZSCH Scientific Instruments Trading, Germany). 5-10 mg of different lyophilized material was tested under an atmosphere of $\mathrm{N} 2$, with test temperature of 30-800 ${ }^{\circ} \mathrm{C}$ and heating rate of $10^{\circ} \mathrm{C} \mathrm{min}^{-1}$.

\subsection{Swelling ratio}

Swelling ratio is the ratio of the mass difference between the swelling gel and the xerogel after a certain time (formula (1)). The lyophilized hydrogels were immersed in deionized water and token out at the specific time points. The surface water of MAC and PMAC hydrogels was dried with filter paper and weighted. The test was stopped when the mass of material remained approximately the same. $W_{\mathrm{t}}$ and $W_{0}$ refer to the mass of gel after water absorption and xerogel, respectively.

$$
\text { Swelling ratio }=\frac{W_{\mathrm{t}}-W_{0}}{W_{0}} \times 100 \%
$$

\subsection{Compression modulus}

The compression modulus is usually determined by a slope of $10 \%$ or $15 \%$ from the stress-strain curve. The module with $10 \mathrm{~mm}$ diameter and $5 \mathrm{~mm}$ high was used to prepare the samples. Different mixture was slowly transferred into the module and the slides were used to cover the module, then the module was placed under UV-irradiation for $30 \mathrm{~min}$. The samples were placed on the Universal Mechanical Tester platform (INSTRON 5967, INSTRON, America) and the upper contact plate was slowly controlled to get close to the sample. The parameter of compression rate was adjusted as 1 $\mathrm{mm} \min ^{-1}$. The test was stopped when the sample was compressed to $60 \%$.

\subsection{Mineralization}

$2 \times$ stimulated body fluid $(\mathrm{SBF})$ was prepared by sequentially dissolving $\mathrm{NaCl}, \mathrm{NaHCO}_{3}, \mathrm{KCl}, \mathrm{K}_{2} \mathrm{HPO}_{4} \cdot 3 \mathrm{H}_{2} \mathrm{O}, \mathrm{MgCl}_{2} \cdot 6 \mathrm{H}_{2} \mathrm{O}$, $\mathrm{CaCl}_{2}$, and $\mathrm{Na}_{2} \mathrm{SO}_{4}$ in distilled deionized water at $37{ }^{\circ} \mathrm{C}$. The MAC and PMAC hydrogels were incubated in $2 \times$ SBF for 7 days, washed three times with deionized water, and then lyophilized. After coating the hydrogels with gold, they were examined by a tungsten filament scanning electron microscope (Thermo Fisher Scientific, USA) at an accelerating voltage of $10 \mathrm{kV}$.

\subsection{Cell culture}

The hFOB cell line (CRL-11372, ATCC, USA) was maintained at $34{ }^{\circ} \mathrm{C}$ in DMEM-F12 containing $10 \%$ foetal bovine serum. The cells were cultured in a humidified atmosphere of $5 \% \mathrm{CO}_{2}$ until confluency was achieved.

\subsection{Live/dead staining}

The live/dead staining reagents PI and AM were used to prepare the original solution at $1: 3$ volume ratio. Every $0.5 \mu \mathrm{L}$ PI solution and $1.5 \mu \mathrm{L} \mathrm{AM}$ solution were added to $1 \mathrm{~mL}$ amicrobic PBS buffer to prepare the working solution. The complete medium in the well of the sample was sucked out and the well 
was washed with amicrobic PBS buffer twice. Then a certain volume of dye solution was added into the well and 48-well plate was covered with silver paper to avoid light under room temperature for $30-45 \mathrm{~min}$. The inverted fluorescence microscope (T1-SM, Nikon, Japan) was used to observe the results.

\subsection{Total protein content}

The proliferation of hFOB cells cultured on the hydrogels were determined by total protein content. After 1,4 and $7 \mathrm{~d}$ of culture, the total proteins were extracted from the cells by lysing in RIPA buffer with a protease inhibitor cocktail (Beyotime Company, China). The protein concentration was determined by a bicinchoninic acid (BCA) protein assay kit (Beyotime).

\subsection{ALP activity}

Alkaline phosphatase (ALP) activity was assayed with Alkaline Phosphatase Assay Kit (P0012S, Beyotime Company, China) according to the manufacturer's instructions. Briefly, cells were washed thrice with PBS and incubated with RIPA Lysis Buffer (P0013K, Beyotime Company, China) for $2 \mathrm{~h}$ in $4{ }^{\circ} \mathrm{C}$ after cultured for 1,4 and 7 d. $50 \mu \mathrm{L}$ lysate of each sample was incubated for $30 \mathrm{~min}$ at $37^{\circ} \mathrm{C}$ in alkaline phosphatase buffer. The reaction was stopped by adding $100 \mu \mathrm{L}$ reaction stop solution and absorbance was measured at $405 \mathrm{~nm}$ on multifunctional microplate reader. A calibration curve with different concentrations of $p$-nitrophenyl was prepared and ALP activity was normalized to the total protein content of each scaffold.

\subsection{Alizarin red S staining}

Mineralization deposition of hFOB cells cultured on scaffolds was determined by alizarin red S Staining. After incubated for 3 and 7 days, cells with adherent mineralized nodules were removed from the scaffolds with trypsin-EDTA and washed with isotonic $\mathrm{NaCl}$. Cells with mineralized nodules were then stained with alizarin red $\mathrm{S}$ for $2 \mathrm{~min}$. After rinsed with ultrapure water to remove the nonspecific stain, the stained nodules were placed in a 96-well plate and observed using a microscopy. Subsequently, alizarin red S staining was extracted by sonicating for $10 \mathrm{~min}$ with $10 \mathrm{mM} \mathrm{HCl}$ in $70 \%$ ethanol. The extracts were then diluted with 5 volumes of PBS and measured at $550 \mathrm{~nm}$ in a multifunctional microplate reader.

\subsection{Statistical analysis}

Values were shown as the means \pm standard error of the mean. All statistical analyses were assessed by one-way or two-way variance (ANOVA). Differences were considered significant when $p<0.05 .0 .01<{ }^{*} p<0.05,0.001<{ }^{* *} p<0.01$ and ${ }^{* * *} p<$ 0.001 .

\section{Results and discussion}

Chitosan is an excellent substrate material in bone tissue engineering, but the modification needs to be done because of the limitation of its physical and chemical properties. In this study, we prepared phosphorylated hydrogel materials by modifying chitosan. The reaction of chitosan with methacrylic anhydride was carried out to prepare methacrylamide chitosan (MAC). Methylallylic anhydride reacted with amino group of side chain of chitosan. Carbon and carbon double bond, which provided the reaction point for subsequent crosslinking reaction, was introduced into chitosan. The photocrosslinking of MAC with different mass fraction of MPC was carried out under the action of photoinitiator. The photoinitiator produced free radicals after irradiation and further initiated the polymerization to prepare PMAC hydrogel.

\subsection{Characterization of MAC}

The synthesis process of MAC is shown in Fig. 1(a). FTIR and ${ }^{1} \mathrm{H}$ NMR was used to compare the structure differences among CS and MAC. As shown in Fig. 1(b), $1613 \mathrm{~cm}^{-1}$ is the characteristic absorption peak of $-\mathrm{NH}_{2}$. Compared to CS, there is an obvious characteristic absorption peak of -NHCO- in $1560 \mathrm{~cm}^{-1}$ which mean the chitosan has been methacryloylated. As shown in Fig. 1(c), olefin double peak shows at 5.3 and $5.6 \mathrm{ppm}$. This means carbon-carbon double bonds of methacrylic anhydride has been successfully grafted on CS. The methacrylation degree of MAC was calculated by comparing the integrated area of H2H6 peaks at 2.8 to $3.9 \mathrm{ppm}$ to that of olefin double peak at 5.3 and $5.6 \mathrm{ppm} .{ }^{30}$ The methacrylation degree of MAC was $17 \%$ using this method.

\subsection{Characterization of PMAC hydrogels}

PMAC hydrogel was prepared as shown in Fig. 2(a). FTIR and ${ }^{31} \mathrm{P}$ NMR was used to compare the structure of MAC and PMAC. In Fig. 2(b), there is an absorption peak of $\mathrm{P}-\mathrm{O}$ in $1000 \mathrm{~cm}^{-1}$. In Fig. 3(c), $0 \mathrm{ppm}$ means primary standard substance of $85 \%$ phosphoric acid. $\pm 50 \mathrm{ppm}$ and $\pm 100 \mathrm{ppm}$ show signal peaks of PMAC 1 and PMAC $0.5, \pm 30$ ppm shows signal peaks of PMAC 0.1 and no signal peak of MAC. The signal peaks are sharper, wider and smoother as the higher mass fraction of MPC in samples. The above signal peaks prove that the PMAC has been successfully bonded with MAC.

\subsection{Properties of PMAC hydrogels}

The TG curve of materials is divided into two parts as shown in Fig. 3(a). The first part is about $30-80{ }^{\circ} \mathrm{C}$ and four groups of materials lost weight due to the loss of absorbed water. In this part, weight loss of MAC, PMAC 1 and PMAC 0.1 are approximately $14 \%$, weight loss of PMAC 0.5 is about $11 \%$. The first stage of TG is material water loss, which contains absorbing water and bind water. PMAC 0.5 has lower weight loss, this may be due to its less absorbing water when it was tested in a drier environment while other three were tested in a rainy day. The second part is about $250-800{ }^{\circ} \mathrm{C}$ and materials lost weight due to the fracture and decomposition of the polymer chains. In this part, the weight loss is approximately $67 \%$ of MAC, approximately $51 \%$ of PMAC 1 and PMAC 0.1 , and approximately $47 \%$ of PMAC 0.5. PMAC materials show a better thermal stability than MAC, yet PMAC 0.5 shows a less weight loss than the other two PMAC materials which could be due to the slightly stronger network structure formed by PMAC 0.5. In the second part of 
(a)
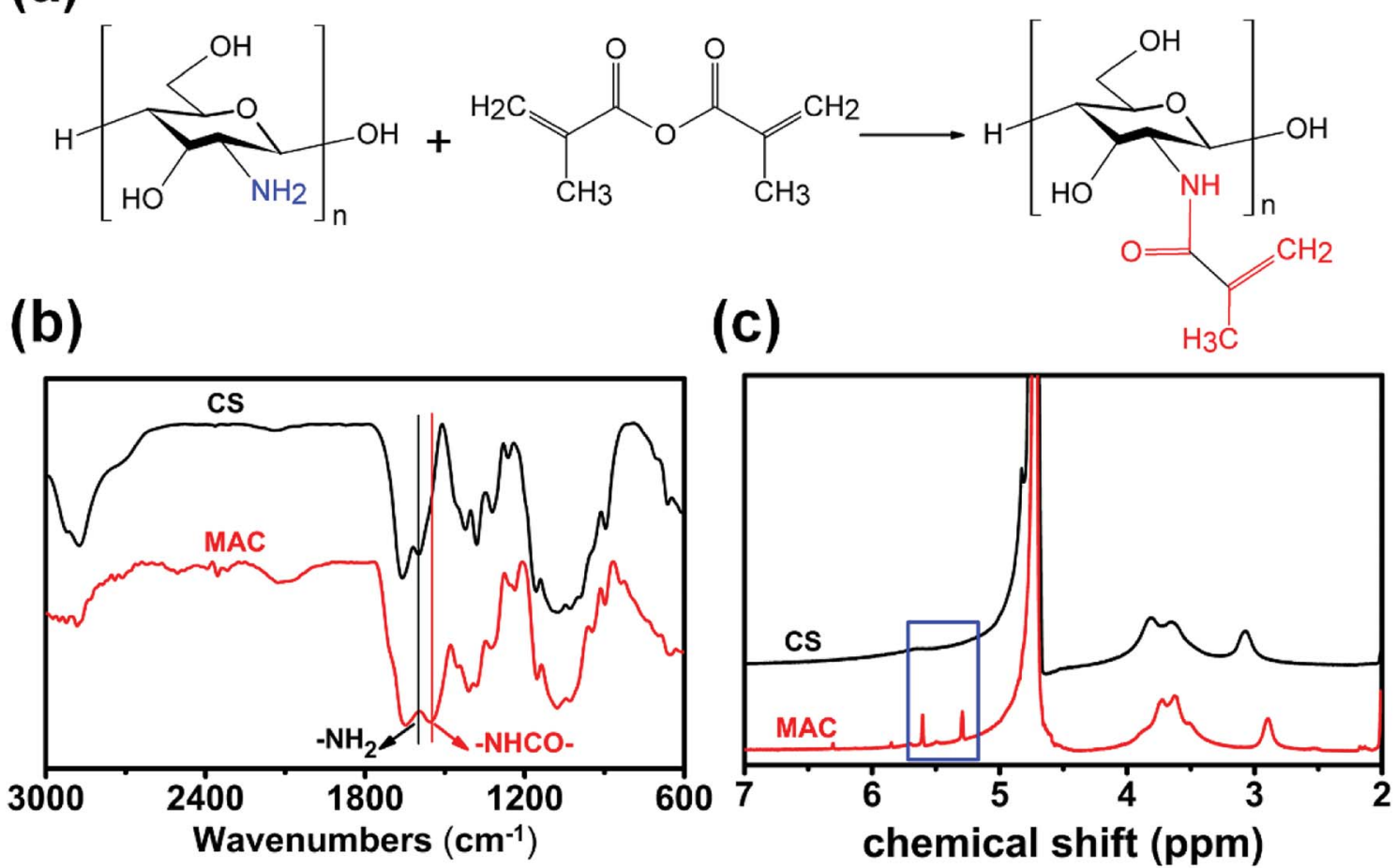

Fig. 1 Synthetic schematic diagram and characterization of MAC. (a) The reaction route of MAC; (b) FTIR results of CS and MAC; (c) ${ }^{1} H$ NMR result of CS and MAC.

TG, PMAC groups decomposed more slowly than MAC and had more solid content in the end. These results indicated that grafting MPC could increase the hydrophilicity and the solubility of MAC, which could facilitate the processability of the material.
To make the scaffold bear the mechanical properties of the originally damaged parts in human body, we increased the cross-linking density of the hydrogels with PMAC. The compression modulus of PMAC groups is significantly higher than that of MAC group as shown in Fig. 3(b). There are (a)
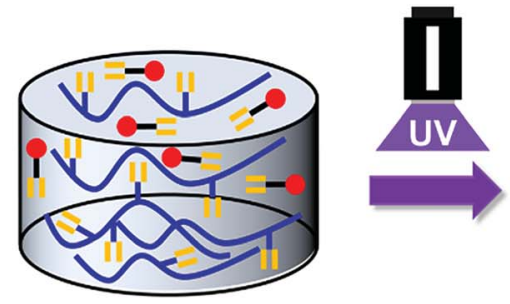

(b)

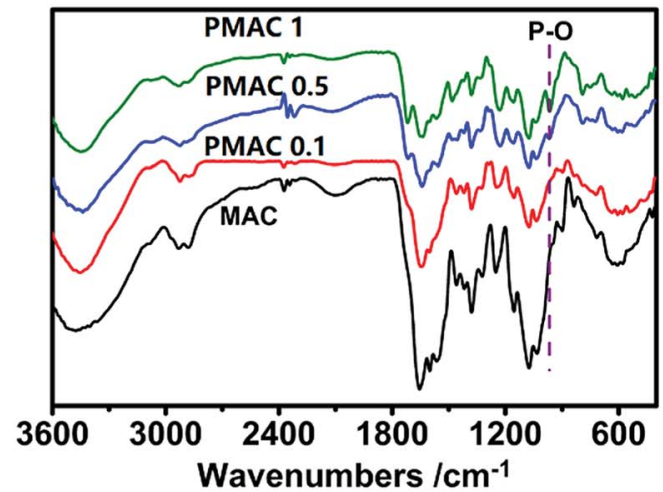

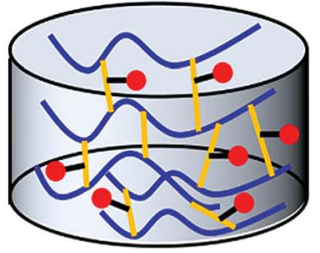

U MAC

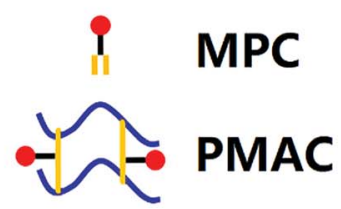

(c)

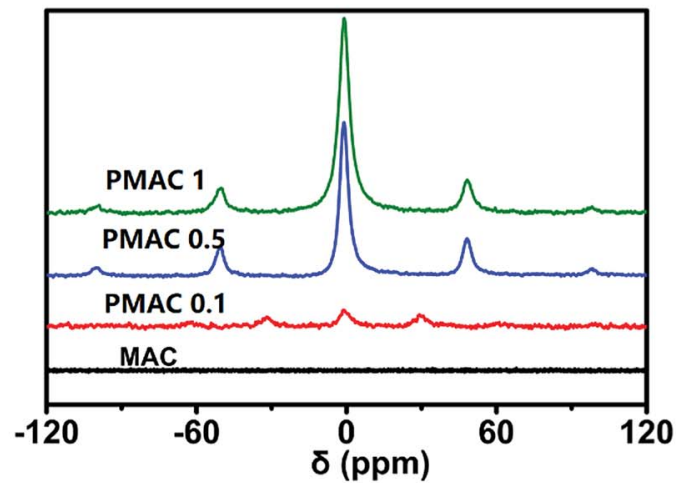

Fig. 2 The schematic of preparation process and characterization of PMAC. (a) Diagrammatic sketch of reaction of PMAC; (b) FTIR result of MAC and different PMAC groups; (c) ${ }^{31} \mathrm{P}$ NMR of MAC and different PMAC groups. 
(a)
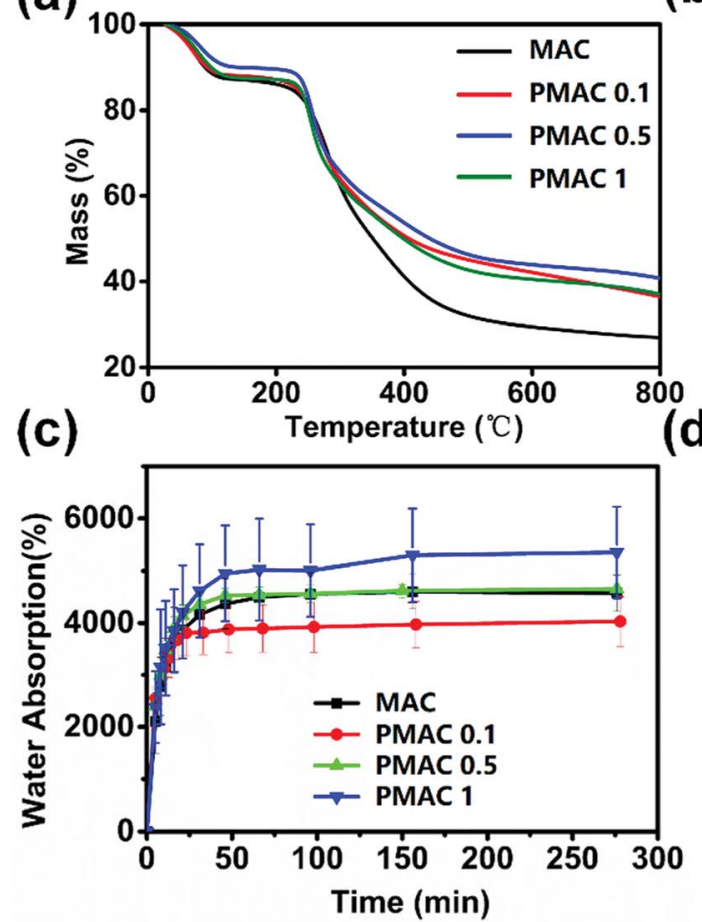

(b) $\widetilde{\pi}$

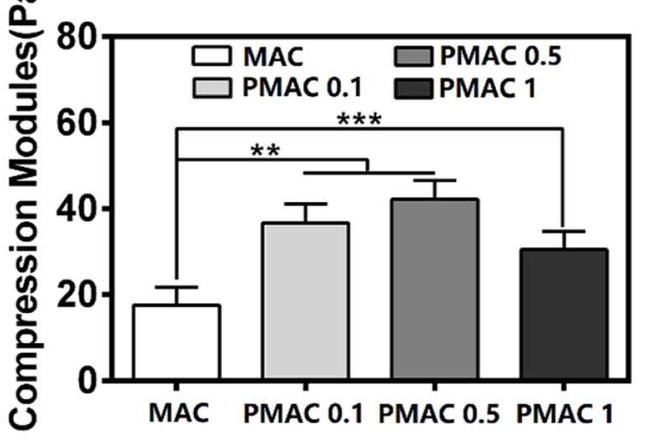

d)
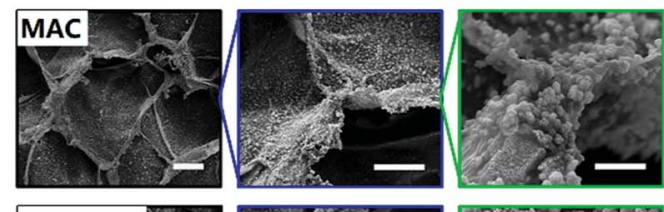

PMAC 0.5
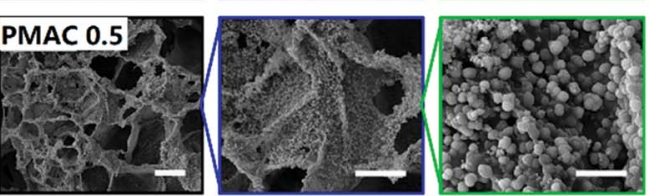

Fig. 3 Properties and morphology of MAC and PMAC. (a) TG curves of MAC and different PMAC groups; (b) compression modules of MAC and different PMAC groups; (c) water absorption of MAC and different PMAC groups; (d) SEM images of MAC and PMAC 0.5, the scale bar were 100, 50 and $10 \mu \mathrm{m}$ from left to right. There was a statistically significant difference between different groups at the same time point $(* p<0.05, * * p<$ $0.01, * * * p 0.001)$.

significant differences among MAC and PMAC groups. Compared with MAC group, the side chain structure of PMAC groups is more complex and the intermolecular connection is closer. Therefore, PMAC groups have better mechanical properties and stronger resistance to deformation.

The swelling ratio of four groups rose rapidly in initial stage and remained stable after about $65 \mathrm{~min}$ as shown in Fig. 3(c). Meanwhile, PMAC hydrogels showed a faster rate than MAC hydrogels in initial stage. The water absorption of MAC, PMAC 1, PMAC 0.5 and PMAC 0.1 is 4677\%, 4089\%, 4653\% and 5610\%, respectively. The MAC and PMAC hydrogels destroyed the intramolecular and intermolecular hydrogen bonds of CS, thus the hydrophilic property of the material was significantly improved. Compared to MAC, PMAC 0.1 contains phosphate groups which could form hydrogen bond with water, thus PMAC 0.1 group show higher water absorption than MAC. However, among three kinds of PMAC hydrogels, high concentration of MPC had a lower water absorption, this may be due to higher MPC dose had a more compact structure that reduced the water absorption of the material. The water absorption test indicated that MAC and PMAC groups had porous structures, which could also be determined in Fig. 3(d). Water absorption ability of our materials and porous structures created a proper environment for cell proliferation, which could be proved in Section 3.4.

Fig. 3(d) shows SEM images of MAC and PMAC 0.5 after incubation in $2 \times \mathrm{SBF}$ for 7 days. The calcium phosphate particles of MAC had irregular shapes with a diameter of about 1.7 $\mu \mathrm{m}$. While calcium phosphate particles of PMAC 0.5 grew as microspheres with a diameter of about $2.5 \mu \mathrm{m}$. Meanwhile, the amount of calcium phosphate particle of PMAC 0.5 hydrogel was obviously more than that of MAC hydrogel. These results show that PMAC hydrogel could accelerate calcium phosphate formation.

\subsection{Cell proliferation}

To evaluate the cytocompatibility of the hydrogels, live/dead staining and total protein content were performed. The phosphorous reagents and photoinitiators used in the preparation of MAC and PMAC hydrogels are low cytotoxic reagents. In Fig. 4(a), staining results of $1 \mathrm{~d}$ have few cells mainly because the cells are not enough when seeded. Nevertheless, the results of 3, 5 and $7 \mathrm{~d}$ show good biocompatibility of MAC and PMAC groups. BCA protein assay was used to determine the proliferation of hFOB cells cultured on the hydrogels. In Fig. 4(b), PMAC groups showed higher protein levels than MAC group. BCA of PMAC 0.5 and PMAC 1 were expressed at higher protein levels than PMAC 0.1, however PMAC 1 showed lower protein level at day 7 than PMAC 0.5. Fig. 4(b) indicated that PMAC groups have no negative effects on cell activity and proliferation and PMAC hydrogels had a better biocompatibility than that of MAC.

\subsection{Osteogenesis}

To investigate the effect of MAC and PMAC hydrogels on osteogenesis, the hFOB cells were cultured on the four groups, 


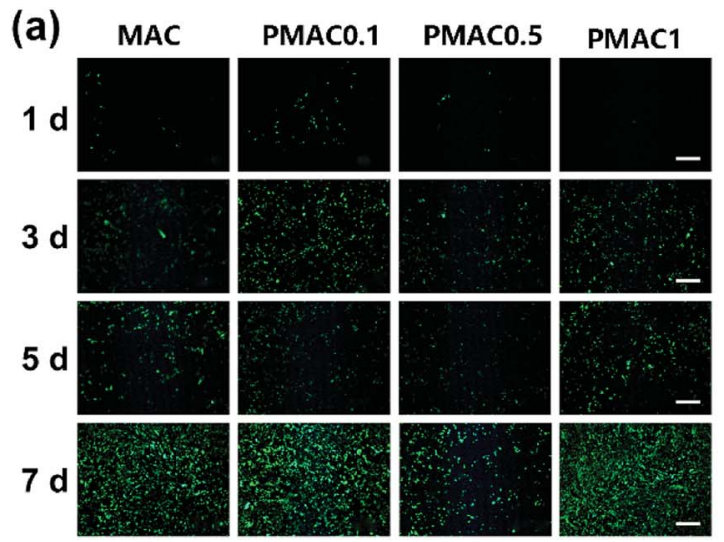

(b)

.

Fig. 4 Proliferation of hFOB cells and BCA protein assay of MAC and PMAC. (a) Live/Dead staining of MAC and different PMAC groups, scale bar was $500 \mu \mathrm{m}$. (b) BCA protein assay of total protein content of MAC and different PMAC groups. There was a statistically significant difference between different groups at the same time point $\left({ }^{*} p<0.05, * * p<0.01,{ }^{* * *} p<0.001\right)$.

ALP was assessed at 1, 4, 7 days and alizarin red S staining were assessed at 3 and 7 days. The calcified areas stained with alizarin red S staining were quantified. As shown in Fig. 5(a), the ALP activity of PMAC 0.1 and PMAC 0.5 was expressed at higher levels than MAC at three time points, and PMAC 0.5 was the highest among three groups. The ALP activity of PMAC 1 was expressed at a higher level at day 4, however with incubation period prolong, the level of ALP activity decreased substantially at day 7. ALP activity showed that PMAC groups could enhance osteogenesis. As shown in Fig. 5(b) and (c), PMAC 0.5 and PMAC 1 groups showed significantly increased formation of calcium deposition and quantified alizarin red S staining of PMAC 1 was (a)

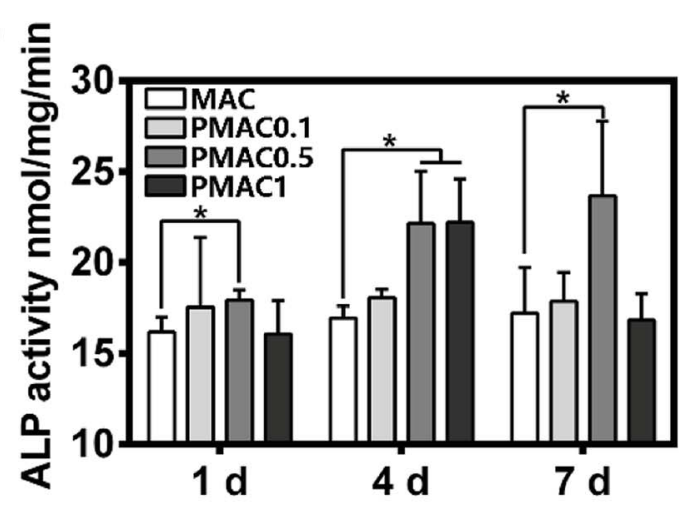

(c)
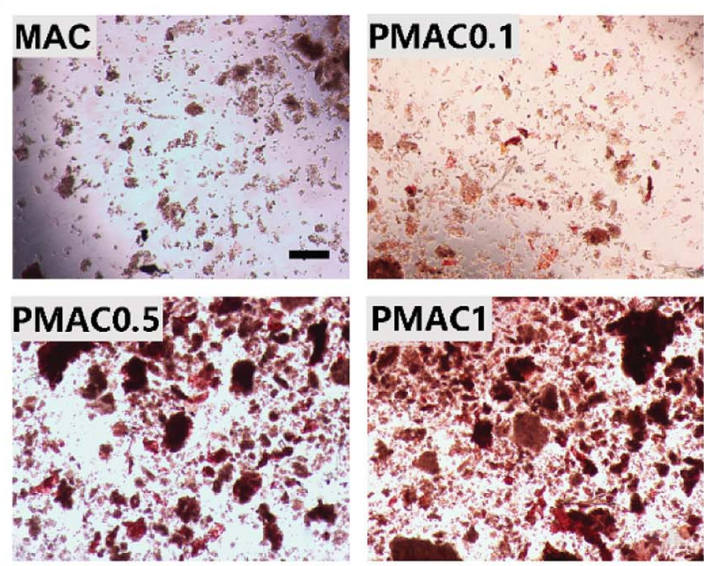

$3 \mathrm{~d}$ (b)
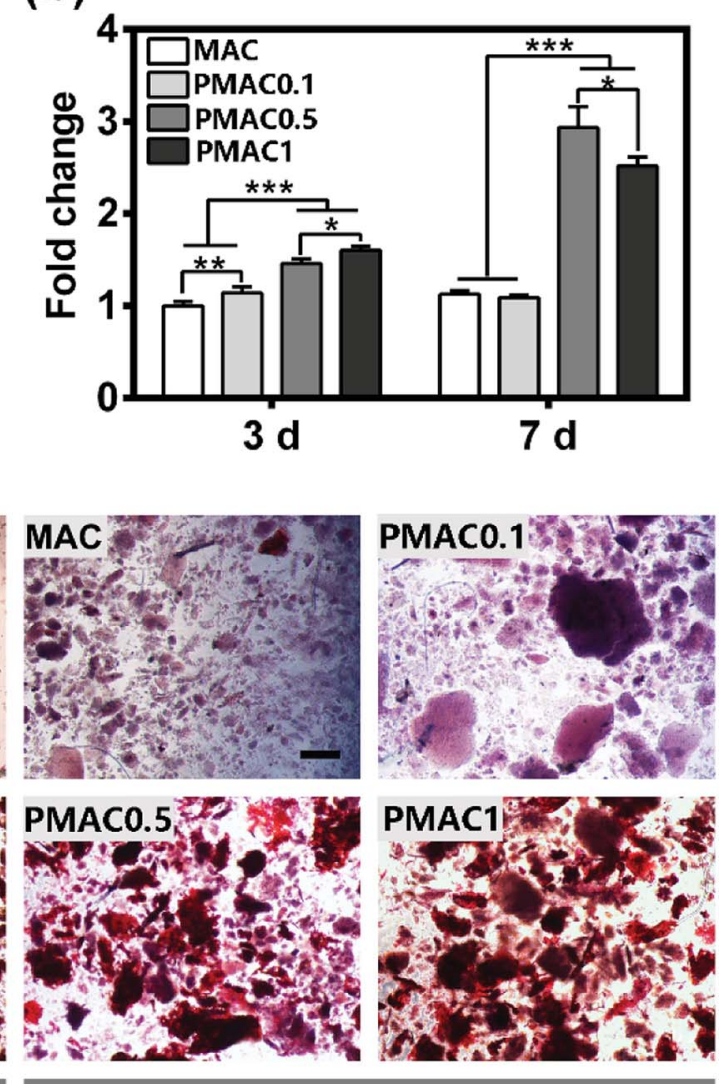

$7 \mathrm{~d}$

Fig. 5 Evaluation of MAC and different PMAC groups on osteogenesis. (a) ALP activity of hFOB cells cultured on MAC and different PMAC groups; (b) quantified alizarin red S staining for hFOB cells; (c) alizarin red S staining for hFOB cells, the scale bar of pictures was $200 \mu \mathrm{m}$. There was a statistically significant difference between different groups at the same time point $(* p<0.05, * * p<0.01, * * * p 0.001)$. 
expressed at a higher level at day 3 while PMAC 0.5 showed a higher level at day 7. Moreover, MAC and PMAC 0.1 were approximately similar low at both day 3 and 7. PMAC groups showed a better capacity on osteogenesis and PMAC 0.5 group showed the most increased calcium deposition. Bone formation is the result of biomineralization in vivo. ALP production is the marker of osteogenic differentiation. PMAC hydrogels had functional $\mathrm{PO}_{4}$ groups, which could increase the nucleation sites thus supporting mineralization compared to the MAC group. The ALP activity of PMAC hydrogels didn't show an increasing trend with the increasing of MPC. This result indicated that PMAC 0.5 might be the most proper dose for osteogenesis compared to the other two PMAC groups.

\section{Conclusion}

In this study, a phosphorylated methacrylamide chitosan (PMAC) hydrogel was successfully prepared by modifying methacrylamide chitosan (AMC) with a low cytotoxicity phosphorus source-2-methacryloyloxyethyl phosphorylcholine (MPC). By characterizing the MAC and PMAC hydrogels, the structure, biocompatibility and osteogenesis ability were confirmed. The PMAC hydrogels had no negative effects on the proliferation of hFOB cells, meanwhile PMAC hydrogels showed better osteogenesis than MAC hydrogels. The PMAC hydrogels have great potential in bone tissue engineering.

\section{Conflicts of interest}

There are no conflicts to declare.

\section{Acknowledgements}

We gratefully acknowledge funding from Shenzhen Science and Technology Program (JCYJ20170306141716014, JCYJ20170815153105076), the National Nature Science Foundation of China (51502095) and Guangdong Natural Science Funds for Distinguished Young Scholars (2016A030306018).

\section{References}

1 M. M. Stevens and J. H. George, Science, 2005, 310, 11351138.

2 Y. C. Chai, A. Carlier, J. Bolander, S. J. Roberts, L. Geris, J. Schrooten, H. Van Oosterwyck and F. P. Luyten, Acta Biomater., 2012, 8, 3876-3887.

3 K. A. Conrads, M. Yi, K. A. Simpson, D. A. Lucas, C. E. Camalier, L. R. Yu, T. D. Veenstra, R. M. Stephens, T. P. Conrads and G. R. Beck Jr, Mol. Cell. Proteomics, 2005, 4, 1284-1296.

4 G. R. Beck Jr, J. Cell. Biochem., 2003, 90, 234-243.

5 G. R. Beck Jr and N. Knecht, J. Biol. Chem., 2003, 278, 4192141929.

6 M. Julien, S. Khoshniat, A. Lacreusette, M. Gatius, A. Bozec, E. F. Wagner, Y. Wittrant, M. Masson, P. Weiss, L. Beck, D. Magne and J. Guicheux, J. Bone Miner. Res., 2009, 24, 1856-1868.
7 A. Jain, A. Karadag, B. Fohr, L. W. Fisher and N. S. Fedarko, J. Biol. Chem., 2002, 277, 13700-13708.

8 R. F. Loese, Exp. Cell Res., 1994, 211, 17-23.

9 A. Veis, C. Sfeir and C. B. Wu, Crit. Rev. Oral Biol. Med., 1997, 8, 360-379.

10 G. He, A. Ramachandran, T. Dahl, S. George, D. Schultz, D. Cookson, A. Veis and A. George, J. Biol. Chem., 2005, 280, 33109-33114.

11 W. J. Landis, K. J. Hodgens, M. J. Song, J. Aren, S. Kiyonag, M. Marko, C. Owen and B. F. McEwen, J. Struct. Biol., 1996, 117, 24-35.

12 K. Li, J. Wang, X. Liu, X. Xiong and H. Liu, Carbohydr. Polym., 2012, 90, 1573-1581.

13 M. Dadsetan, M. Giuliani, F. Wanivenhaus, M. Brett Runge, J. E. Charlesworth and M. J. Yaszemski, Acta Biomater., 2012, 8, 1430-1439.

14 Y. K. Kim, L. S. Gu, T. E. Bryan, J. R. Kim, L. Chen, Y. Liu, J. C. Yoon, L. Breschi, D. H. Pashley and F. R. Tay, Biomaterials, 2010, 31, 6618-6627.

15 P. Huang, X. Bi, J. Gao, L. Sun, S. Wang, S. Chen, X. Fan, Z. You and Y. Wang, J. Mater. Chem. B, 2016, 4, 2090-2101. 16 D. Wang, Biomaterials, 2003, 24, 3969-3980.

17 B. M. Watson, F. K. Kasper, P. S. Engel and A. G. Mikos, Biomacromolecules, 2014, 15, 1788-1796.

18 I. C. Stancu, R. Filmon, C. Cincu, B. Marculescu, C. Zaharia, Y. Tourmen, M. F. Baslé and D. Chappard, Biomaterials, 2004, 25, 205-213.

19 C. R. Nuttelman, D. S. Benoit, M. C. Tripodi and K. S. Anseth, Biomaterials, 2006, 27, 1377-1386.

20 D. S. Benoit, M. P. Schwartz, A. R. Durney and K. S. Anseth, Nat. Mater., 2008, 7, 816-823.

21 P. Huang, X. P. Bi, J. Gao, L. J. Sun, S. F. Wang, S. Chen, X. Q. Fan, Z. W. You and Y. D. Wang, J. Mater. Chem. B, 2016, 4, 2090-2101.

22 C. Xu, P. Su, X. Chen, Y. Meng, W. Yu, A. P. Xiang and Y. Wang, Biomaterials, 2011, 32, 1051-1058.

23 X. Wang, J. Ma, Q. Feng and F. Cui, Biomaterials, 2002, 23, 4591-4600.

24 P. Datta, S. Dhara and J. Chatterjee, Carbohydr. Polym., 2012, 87, 1354-1362.

25 L. S. Gu, Y. K. Kim, Y. Liu, K. Takahashi, S. Arun, C. E. Wimmer, R. Osorio, J. Q. Ling, S. W. Looney, D. H. Pashley and F. R. Tay, Acta Biomater., 2011, 7, 268-277.

26 J. Tan, R. A. Gemeinhart, M. Ma and W. M. Saltzman, Biomaterials, 2005, 26, 3663-3671.

27 P. M. Lopez-Perez, R. M. da Silva, R. A. Sousa, I. Pashkuleva and R. L. Reis, Acta Biomater., 2010, 6, 3704-3712.

28 Y. Iwasaki and K. Ishihara, Sci. Technol. Adv. Mater., 2012, 13, 064101.

29 K. Ishihara, Colloids Surf., B, 2000, 18, 325-335.

30 L. M. Yu, K. Kazazian and M. S. Shoichet, J. Biomed. Mater. Res., Part A, 2007, 82, 243-255.

31 M. Lavertu, Z. Xia, A. N. Serreqi, M. Berrada, A. Rodrigues, D. Wang, M. D. Buschmann and A. Gupta, J. Pharm. Biomed. Anal., 2003, 32, 1149-1158. 